\title{
Primary infection protects pigs against re-infection with Lawsonia intracellularis in experimental challenge studies
}

Riber, Ulla; Hvass, Henriette Cordes; Boutrup, Torsten Snogdal; Jensen, Tim Kåre; Heegaard, Peter M. H.; Jungersen, Gregers

Published in:

Veterinary Microbiology

Link to article, DOI:

10.1016/j.vetmic.2010.11.028

Publication date:

2011

Link back to DTU Orbit

Citation (APA):

Riber, U., Hvass, H. C., Boutrup, T. S., Jensen, T. K., Heegaard, P. M. H., \& Jungersen, G. (2011). Primary infection protects pigs against re-infection with Lawsonia intracellularis in experimental challenge studies. Veterinary Microbiology, 149(3-4), 406-414. https://doi.org/10.1016/j.vetmic.2010.11.028

\section{General rights}

Copyright and moral rights for the publications made accessible in the public portal are retained by the authors and/or other copyright owners and it is a condition of accessing publications that users recognise and abide by the legal requirements associated with these rights.

- Users may download and print one copy of any publication from the public portal for the purpose of private study or research.

- You may not further distribute the material or use it for any profit-making activity or commercial gain

- You may freely distribute the URL identifying the publication in the public portal 


\title{
Primary infection protects pigs against re-infection with Lawsonia intracellularis in experimental challenge studies
}

\author{
Ulla Riber*, Henriette Cordes, Torsten S. Boutrup, Tim K. Jensen, Peter M.H. Heegaard, \\ Gregers Jungersen \\ National Veterinary Institute, Technical University of Denmark, Bülowsvej 27, 1790 Copenhagen V, Denmark
}

\section{A R T I C L E I N F O}

\section{Article history:}

Received 1 July 2010

Received in revised form 15 November 2010 Accepted 19 November 2010

\section{Keywords:}

Lawsonia intracellularis

Re-infection

Protection

Acute phase protein response

IHC

Pathology

\begin{abstract}
A B S T R A C T
In two separate trials pigs were experimentally infected with Lawsonia intracellularis at 56 weeks of age followed by antibiotic treatment and resolution of the primary infection and then re-inoculated at 12-13 weeks of age. A treatment-control group of pigs received the primary infection and antibiotic treatment only, and served as control for the antibiotic treatment of the primary infection. A challenge-control group of pigs received the second inoculation dose only at 12-13 weeks of age to control infectivity of the challenge-dose and susceptibility of pigs to L. intracellularis at this age. Pigs were monitored for shedding of $L$. intracellularis in faeces by PCR, and for the development of antibodies and responses of acute phase proteins in serum. The presence of $L$. intracellularis antigen in the intestinal mucosa was examined in post mortem samples by immunohistochemistry.

In both trials primary infected pigs were protected from infection after challenge inoculation as evidenced by absence of faecal shedding of $L$. intracellularis, lack of changes in acute phase protein concentrations after challenge and with low levels of bacterial antigen in the intestinal mucosa of re-inoculated pigs comparable to that of the treatmentcontrol pigs. In contrast, challenge-control pigs shed $L$. intracellularis in faeces, had $L$. intracellularis antigen extensively present within all layers of the intestinal mucosa and developed a significant acute phase protein response in serum after the experimental infection. The acute phase protein response to $L$. intracellularis infection was detected as an increased rise in the serum concentrations of C-reactive protein and haptoglobin from day- 6 post infection, and increased serum concentrations of haptoglobin were generally seen 2-3 weeks after inoculation both at 5-6 and 12-13 weeks of age.

In conclusion substantial protection against $L$. intracellularis infection was found in the re-inoculated pigs in contrast to the development of infection in age-matched control pigs. The acute phase protein responses reflected both the observed protection against $L$. intracellularis infection upon secondary challenge and that increased resistance to the infection develops with age.
\end{abstract}

(c) 2010 Elsevier B.V. All rights reserved.

\section{Introduction}

Lawsonia intracellularis is an obligate intracellular bacterium that causes proliferative enteropathy (PE) in pigs. The infection is common worldwide, and in Denmark

\footnotetext{
* Corresponding author. Tel.: +45 35886233

E-mail address: urib@vet.dtu.dk (U. Riber).
}

L. intracellularis can be detected in most pig herds. However, infection with $L$. intracellularis does not always cause disease. Generally, weaned pigs and growing pigs develop disease and clinical signs include diarrhoea, uneven weight gain and poor performance. In pigs more than 12 weeks old the infection can cause a more acute hemorrhagic diarrhoea and sudden death (McOrist and Gebhart, 2006). At herd-level PE is mainly controlled by antibiotic treatment and vaccination with an attenuated 
live oral vaccine Enterisol ${ }^{\circledR}$ Ileitis vet., Boehringer Ingelheim (Jacobson et al., 2010). The economic losses caused by PE due to uneven weight gain, mortality, medication and/ or vaccination are high (McOrist, 2005).

Ability to induce protection against $L$. intracellularis infection has been investigated by oral administration of the vaccine strain (Kroll et al., 2004), in a field evaluation of the L. intracellularis vaccine (McOrist and Smits, 2007) and in a re-infection study by Collins and Love (2007). Some protection was shown by reduced faecal shedding of L. intracellularis and reduced scores of specific lesions at post mortem examination in these studies. However, in the study by Collins and Love (2007) age matched pigs were not used to control infectivity of challenge inoculation.

The overall aim of the present study was to investigate protection against $L$. intracellularis infection in age matched pigs using an experimental re-infection model. In two independent experimental infection trials we found a substantial and reproducible protection against challenge infection with $L$. intracellularis compared to development of infection with shedding of bacteria and significant acute phase protein response in serum of age-matched pigs receiving a primary infection. The acute phase protein response is an unspecific indicator of disease and can be measured as an increase in serum concentration of e.g. haptoglobin and C-reactive protein (CRP) in pigs (Petersen et al., 2004). To our knowledge the current data are the first to demonstrate an acute phase protein response to $L$. intracellularis infection and disease.

\section{Materials and methods}

\subsection{Animals and experimental design}

A total of 62 pigs (Danish Landrace/Yorkshire/Duroc) of mixed sex were used. The pigs used in Experiment I and II, respectively, came from two different high health and SPF herds both negative for $L$. intracellularis after a medicated eradication program as shown by routine diagnostic testing on faecal samples by PCR and serum samples by ELISA. Additionally the herds were negative for Salmonella according to the Danish surveillance program and Brachyspira hyodysenteriae, Mycoplasma hyopneumoniae, Actinobacillus pleuropneumoniae, toxin-producing Pasteurella multocida, PRRS virus, lice and manges according to the
SPF program. Cases of other infections in the herds that could interfere with the experiments were not stated.

At 4-5 weeks of age (weight $8-12 \mathrm{~kg}$ ) pigs were randomly grouped and housed in separate and isolated pens with concrete slatted floor, straw bedding and ad libitum access to water. Pigs were fed standard pelleted growing feed, and were allowed to acclimatize for one week before starting the experiment. During this period faeces and blood were collected, and PCR and ELISA diagnostic testing confirmed the absence of $L$. intracellularis infection. Two separate experiments (I and II) with similar experimental designs were performed, however, size of inoculum, number of samplings and end-point of the experiments were different. A schematic overview is shown in Table 1.

Experimental infections with $L$. intracellularis were induced by an oral challenge model, using L. intracellularis infected intestinal mucosa isolate from pigs with PE (Boesen et al., 2004). Age-matched non-inoculated pigs served as negative controls.

Pigs in the Re-infection (Re-i) and Treatment Control (TC) groups were inoculated with $L$. intracellularis at day 0 , while pigs in the Challenge Control (CC) group were uninfected. At day 21 all pigs in all groups were treated with tiamulin (Tiamutin ${ }^{\circledR}$ Vet.) to clear the L. intracellularis infection. Tiamulin was administrated in the feed $(20 \mathrm{mg}$ tiamulin/g) for 7 days in both experiments and was in Experiment I preceded by a 3-day intra muscular injection treatment $(20 \mathrm{mg} / \mathrm{kg}$ pig) to treat clinical infection. Uninfected control pigs also received the antibiotic treatment in order to equal any effects related to this treatment. In the beginning and at the end of the treatment period the pens were cleaned thoroughly with highpressure water and subsequently disinfected with a broad spectrum disinfectant ( $2 \%$ solution of Virkon ${ }^{\circledR}$ S, Antec International Limited, Suffolk, UK).

Following a three-week resolving phase after antibiotic treatment, a challenge $L$. intracellularis inoculation was given to pigs in the Re-i and CC groups. Thus, TC pigs acted as controls for effective antibiotic treatment against the primary inoculation and CC pigs were age-matched controls for the challenge inoculation.

In Experiment I, all pigs were killed at day 70/74 (approx. three weeks after challenge). In this experiment, one pig died (day 7), one pig was euthanized and

Table 1

Schematic overview of the experimental design.

Day: 0 Primary infection Antibiotic


Table 2

Group identity and number of pigs in the groups.

\begin{tabular}{llll}
\hline Group & Code and identification in figures & Final number of pigs & Experiment I \\
\cline { 2 - 4 } & & Ee-i & 8 \\
Re-infection & $\mathrm{CC}$ & 10 \\
Challenge control & $\mathrm{TC}$ & 4 & 10 \\
\hline
\end{tabular}

Numbers of pigs in each group at the end of the experiments. In addition nine L. intracellularis inoculated pigs and five non-inoculated control pigs were killed during the experiment (Experiment II, day 19/20), data from these pigs are included in the respective groups until death. Furthermore three pigs from Experiment I were excluded from the whole experiment and one pig from Experiment II was excluded from day 49 and forward. For details see Section 2.1. Codes used for identification of pig-groups in the paper are shown in the table. Before day 48 , time of challenge inoculation, pigs were grouped together as $L$. intracellularis inoculated pigs ( $\bullet$ and non-inoculated control pigs $(\bigcirc)$.

necropsied (day 55) because of no weight gain, and one pig revealed chronic fibrinous polyserositis unrelated to $L$. intracellularis infection at necropsy at the end of the experiment. These three pigs were excluded from the whole experiment. In Experiment II, nine primary inoculated pigs and 5 non-infected control pigs were killed (at day 19/20) nearly three weeks after inoculation to measure extent of early onset of $L$. intracellularis infection after the primary infection. The rest of the pigs were killed at day 83/84 (five weeks after challenge). In this experiment one CC-pig died at day 54 due to non-intestinal disorder (acute interstitial pneumonia). Faecal and blood parameters of this pig until death are included in the study (Table 2).

The experiments were conducted in accordance with the guidelines laid down by the Danish Animal Experiments Inspectorate under the Ministry of Justice.

\subsection{Preparation of inoculum and inoculation of pigs}

Inoculation material was prepared from frozen $\left(-80^{\circ} \mathrm{C}\right)$ severely PE affected porcine small intestines as previously described (Boesen et al., 2004). The concentration of $L$. intracellularis in the inoculum was quantified by indirect immuno-fluorescence as described by Jensen et al. (1997) using an anti-L. intracellularis monoclonal antibody (Law1DK) (Boesen et al., 2005a).

In Experiment I, pigs were fasted from the previous evening and inoculated by oro-gastric tube with $25 \mathrm{ml}$ of inoculum. The pigs received approximately $10^{10} \mathrm{~L}$. intracellularis per pig at day 0 and at day 49 .

In Experiment II, pigs were fasted from the previous evening and inoculated per oral (allowing the pigs to swallow the inoculum) with $2 \mathrm{ml}$ of inoculum at day 0 , approximately $2 \times 10^{9}$ L. intracellularis per pig, and $10 \mathrm{ml}$ at day 49 , approximately $10^{10} \mathrm{~L}$. intracellularis per pig.

The intestinal material used for both experiments was from same batch of small intestines collected from pigs highly infected with $L$. intracellularis. The pigs originated from a Danish fattening herd with low Salmonella prevalence according to the Danish Salmonella surveillance program. A moderate level of porcine circovirus type 2 (PCV-2) DNA copies were found in the inoculum by PCR (Hjulsager et al., 2009; Jensen et al., 2006), but no differences in anti-PCV-2 titer in serum pre- and post inoculation could be detected in the pigs (data not shown).

\subsection{Weight gain and clinical observation}

The pigs were weighed at arrival, after the first infection phase, before and three weeks into the second infection phase and at the end of experiment.

Daily observation of performance and faeces fluidity was performed and individual observations that were different from normal observations in healthy pigs were noted.

\subsection{Bacteriological examinations}

Faecal samples were collected with regular intervals and measured for $L$. intracellularis by real time PCR (Lindecrona et al., 2002). The DNA extract from the faeces samples were diluted 1:100 in Milli-Q water and stored at $4{ }^{\circ} \mathrm{C}$ until further analysis. Samples of the extracts were run on a RotorGene 3000 (Qiagen, Copenhagen, Denmark) using primers and probes based on the 16S rDNA of $L$. intracellularis.

\subsection{Necropsy and immunohistochemical examination}

Necropsy was performed around three weeks and five weeks after $L$. intracellularis inoculation. All pigs were subject to necropsy with special emphasis to describe the extent of proliferative enteropathy (PE) and to exclude pigs with other lesions that might have influenced the experimental infection study.

Tissue samples were taken from jejunum (only in Experiment I), ileum, colon and the ileal lymph node for immunohistochemical (IHC) detection of $L$. intracellularis antigenic material using $\mathrm{mAb}$ Law1-DK according to Boesen et al. (2004). Blinded samples were scored according to the severity and extension of the lesions: (0) no detection of antigen, (1) focal, (2) moderate, and (3) extensive detection of $L$. intracellularis antigen in epithelium and lamina propria, respectively.

\subsection{Measurement of acute phase proteins}

Haptoglobin was analysed by a sandwich ELISA using the monoclonal antibody 3.8D7 directed against porcine haptoglobin as described by Sørensen et al. (2006). This ELISA was calibrated against a porcine haptoglobin standard (Saikin Kagaku Co., Ltd, Japan), using a pooled 
porcine serum as a secondary standard on all plates. Standard and samples were run in duplicate and the detection limit was $3.3 \mu \mathrm{g} / \mathrm{ml}$. CRP was determined by a modified version of the sandwich ELISA described by Eckersall et al. (1989), employing a phosphoryl choline coupled dendrimer as the catching-layer and anti human CRP (A0073, DAKO, Glostrup, Denmark) as the detection antibody in a sandwich ELISA (Heegaard et al., 2009). As inplate standard was used a porcine serum pool previously calibrated using a human serum C-reactive protein calibrator (X0923, DAKO). Standards and samples were run in duplicate and the detection limit was $0.2 \mu \mathrm{g} / \mathrm{ml}$.

\subsection{Measurement of serological response}

The specific IgG responses to $L$. intracellularis were measured using deoxycholate extracted antigen in ELISA as described by Boesen et al. (2005b). A cut off at 15 OD\% for the present study was established by frequency analysis (95\% negative) of the non-inoculated pigs from eight weeks of age and older.

\subsection{White blood cell count}

Alterations in white blood cell (WBC) count in blood samples were measured in fresh heparin stabilized blood samples by automatic counting using AC900, Swelab (Experiment $\mathrm{I}$ ) or $\mathrm{ABC}$ Vet (Vet $\mathrm{ABC} \mathrm{TM}^{\mathrm{TM}}, \mathrm{ABX}$, Montpellier, France) (Experiment II) haematology instruments.

\subsection{Statistical analyses}

Mann-Whitney test was used to test differences between pig groups and between responses on different days. Two way ANOVA with Bonferroni post test was used to compare acute phase protein response in L. intracellularis-inoculated pigs and non-inoculated pigs. Student's ttest was used to compare weight gain between different groups of pigs after inoculations.

\section{Results}

\subsection{Clinical signs}

In Experiment I, clinically signs, with depressed behaviour, reduced food consumption and diarrhoea (semi-solid to watery stools) were observed in four pigs (\#3, \#11, \#19, \#22) out of 12 pigs during week 2-3 after primary inoculation with approx. $10^{10}$ L. intracellularis per pig at approx. 5-6 weeks of age. The other inoculated pigs showed only subclinical signs of disease with soft faeces, but with normal behaviour. In Experiment II, only subclinical signs of disease (soft faeces, but normal behaviour) were observed in three pigs (\#27, \#37, \#52) out of 24 pigs after primary inoculation with approx. $10^{9} \mathrm{~L}$. intracellularis per pig while all other pigs were without any signs of disease.

After challenge inoculation with $10^{10}$ bacteria per pig at approx. 12-13 weeks of age, no signs of clinical or subclinical disease were observed in any of the two experiments in neither the re-inoculated nor the challenge control pigs.

\subsection{Faecal shedding}

Results of faecal shedding are presented in Fig. 1. During 3-4 weeks after primary $L$. intracellularis inoculation at day 0 , at least one PCR positive faecal sample was detected in all pigs in Experiment I, and in all but one (\#43) of the pigs in Experiment II. Among the nine $L$. intracellularis inoculated-pigs killed after three weeks (day 19/20 p.i.), L. intracellularis was not detected in faecal samples in four (\#40, \#50, \#60,\#63) of these pigs, however L. intracellularis was detected by IHC at necropsy (Fig. 2B). In both experiments the antibiotic treatment stopped the faecal shedding of $L$. intracellularis and the TC group stayed PCR negative for $L$. intracellularis for the rest of the experiment. After challenge inoculation, faecal shedding of
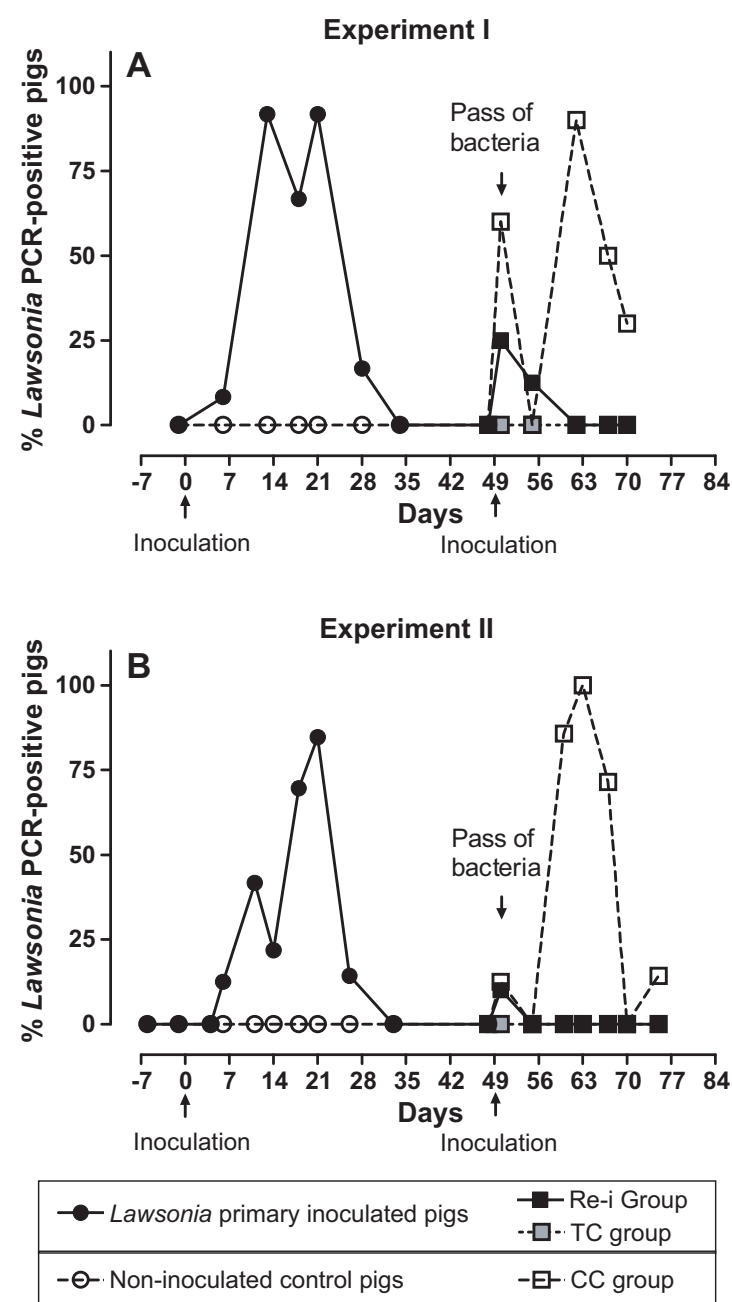

Fig. 1. PCR-detection of $L$. intracellularis in faecal samples. L. intracellularis was measured in individual faecal samples and the ratio of pigs with positive $L$. intracellularis detection was calculated. Before day 48, the TCpigs and the Re-i pigs were grouped together $(\mathbf{0})$ as pigs receiving primary L. intracellularis infection, the pigs in the CC-group were in this period non-inoculated control pigs (O). In Experiment II, data from the nine L. intracellularis infected and five non-inoculated control pigs, killed at day 19/20, were included from day -7 to day 18 . 
Experiment I: A

IHC examination 3 weeks after challenge inoculation

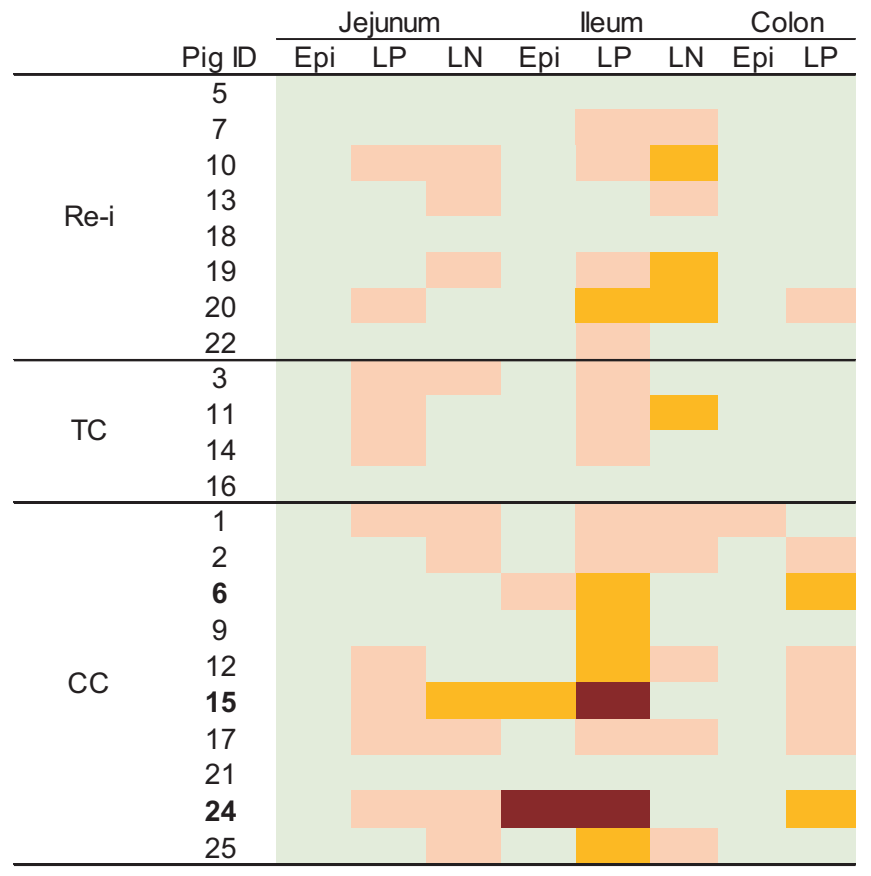

Experiment II: B

IHC examination 3 weeks after primary inoculation

\begin{tabular}{|c|c|c|c|c|c|}
\hline & & lleum & Mes. & & \\
\hline & Pig ID & $\begin{array}{ll}\text { Epi } & \text { LP }\end{array}$ & $\mathrm{LN}$ & Epi & LP \\
\hline No & All pigs & & & & \\
\hline inoculation & $\mathrm{N}=5$ & & & & \\
\hline & 40 & & & & \\
\hline & 60 & & & & \\
\hline & 69 & & & & \\
\hline & 34 & & & & \\
\hline inculation & 63 & & & & \\
\hline & 65 & & & & \\
\hline & 32 & & & & \\
\hline & 45 & & & & \\
\hline & 50 & & & & \\
\hline
\end{tabular}

Experiment II: $\mathrm{C}$

IHC examination 5 weeks after challenge inoculation

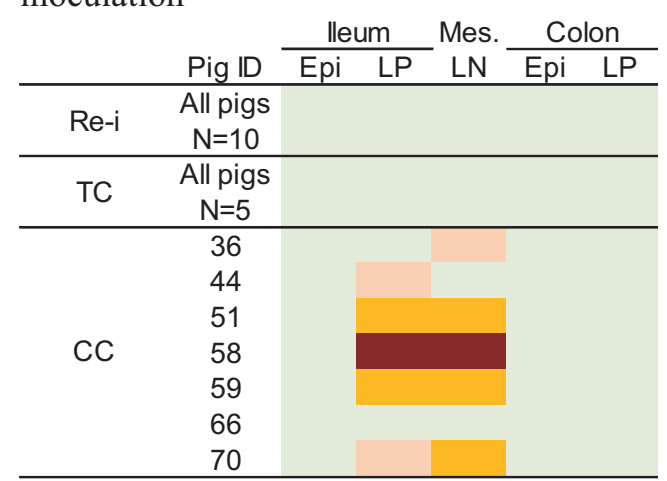

Fig. 2. Scores from immunohistochemical examination for $L$. intracellularis antigen in intestinal tissues. The scoring of the intestines was recorded for epithelium (Epi) and lamina propria (LP) and lymph nodes (LN) separately according to severity and extension of lesions: No staining $(=0)$, focal $(=1)$, moderate ( $(=2)$, and extensive detection $(=3)$ of $L$. intracellularis antigen. The identities of individual pigs (Pig ID) in the groups are partly shown in the tables. Pig ID in bold (Experiment I: 6, 15, 24) gross examination revealed PE. The ID of pigs in the group No inoculation (Experiment II, 3 weeks) is: 31,42 , 47, 49, and 67. For Experiment II (5 weeks) the ID of pigs is: Re-I group: 26, 37, 38, 52, 53, 55, 57, 61, 64, and TC group: 27, 29, 43, 62, and 68. (For interpretation of the references to color in this figure legend, the reader is referred to the web version of the article.)

L. intracellularis was detected in all but one (\#21, Experiment I) of the 18 pigs in the challenge control groups in the two experiments. In contrast, faecal shedding of $L$. intracellularis could not be detected in any pigs of the Re-i group. An initial pass of $L$. intracellularis from the inoculum was observed on day 50, the day after challenge inoculation, in both re-infection and challenge control groups.

\subsection{Weight gain}

In both experiments, some pigs (\#3, \#11, \#16, \#19, \#20 and \#27, \#37, \#52) showed reduced weight gain during the primary infection compared to the other pigs. For Experiment I this difference was statistically significant $(P<0.05$, student's $t$-test) when comparing the primary infected pigs with the uninfected CC pigs from day 0-21. In 
Experiment II, where the primary inoculation only resulted in subclinical disease, the statistical difference in mean weight gain from start of experiment until day 21 was not significant $(P=0.123)$. Likewise, comparing mean daily weight gain over the whole study period was not statistically significant between groups in either of the experiments.

\subsection{Pathological and immunohistochemical examination}

In Experiment I, gross examination revealed PE in 3/10 pigs from the CC group, indicating that the 12 weeks old pigs were susceptible for $L$. intracellularis infection, whereas in the Re-i and TC pigs only minor affections with enteritis or normal appearance without signs of infection was found. Although L. intracellularis antigen was detected in the intestines by IHC in nearly all pigs of Experiment I (Fig. 2A) three weeks after challenge inoculation the CC group showed significantly higher total score $(P=0.0316$, Mann-Whitney) compared to the Re-i group and TC group, which was at a lower but similar level. In Experiment II, L. intracellularis antigen was not detected in any pigs in the Re-i group and TC group, five weeks after challenge inoculation, whereas L. intracellularis was found in 6/7 pigs in the CC group mainly in lamina propria of ileum and in the ileal lymph node, but not in colon (Fig. 2C). Histopathologically, signs of enteritis related to other infections than $L$. intracellularis were not observed.

\subsection{Acute phase protein response}

Experiment I: During the first infection period haptoglobin concentrations were significantly higher $(P=0.0001)$ in the group of $L$. intracellularis inoculated pigs compared to the uninfected controls (Fig. $3 \mathrm{~A}$ ). In the uninfected control group a drop in haptoglobin concentration from day -1 to day 6 was observed, presumably indicating accustomization of these animals during this period. In contrast, a substantial rise in serum concentration of haptoglobin was detected at day 13 and persisting to day 21 in the $L$. intracellularis primary infected group. After antibiotic treatment and resolution of the infection both groups converge to an intermediate level (day 41) where after the primary infected group stayed at this level or dropped further at some time points after challenge inoculation (from day 49, Re-i group), showing the same concentrations of haptoglobin as the TC group not receiving challenge inoculation. By contrast, the CC group, showed a rise in serum concentration of haptoglobin after challenge with significantly higher concentrations at day 60 (day 11 p.i.) $(P<0.01$, Mann-Whitney) compared to the Re-i group. C-reactive protein (CRP) showed the same general changes, although the rise after primary infection occurs already at day 6 and there was a less pronounced drop in the non-infected group during the first period. Also, there was less of a rise in the challenge control group after challenge inoculation at day 49 (Fig. 3B). Neither serum concentrations of haptoglobin nor CRP was significantly increased upon challenge inoculation in the Re-i group (Fig. 3A and B).
Experiment II (Fig. 3C): As observed in Experiment I, an increase in serum haptoglobin concentrations was seen (especially at day 11 and/or 14 in most pigs) in response to primary inoculation with $L$. intracellularis, followed by a decrease. In the same period the haptoglobin concentrations decreased in the group of non-inoculated control pigs. An unexplained slight increase at day 6 was measured in some control pigs. After antibiotic treatment and resolving of the infection, the concentrations of haptoglobin stayed at low level in $4 / 5$ pigs in the TC group until the end of experiment. The serum concentrations of haptoglobin in the Re-i group after challenge inoculation at day 49 were not significantly different from the concentrations in the TC group, however 4/10 pigs (\#37, \#38, \#55, \#53) in the Re-i group showed a 2-3 fold higher haptoglobin concentration at day 60 compared to before re-inoculation. In contrast, following inoculation at day 49 the CC group (all eight pigs) experienced increased serum concentrations of haptoglobin, and, compared to the Re-i group, with significantly higher haptoglobin concentrations (MannWhitney $P<0.005$ at day 55-82, except day $60 P=0.19$ ). $\mathrm{CRP}$ responses were not analysed in Experiment II.

\subsection{L. intracellularis serum antibodies}

In Experiment I, all serum samples taken before primary inoculation and of CC group during the 7 weeks before their challenge inoculation, i.e. from the noninoculated pigs, were sero-negative. In contrast, nearly half of the pigs (45.6\%) from Experiment II were ELISA-positive prior to the primary L. intracellularis inoculation. None of these pigs were shedding L. intracellularis in faeces, and by day 25 (approximately at 8 weeks of age) all the noninoculated CC pigs were ELISA-negative indicating that these reactions were caused by maternal antibodies.

In response to the primary $L$. intracellularis infection, 7 of 12 pigs (58.3\%) in Experiment I and 9 of 13 (69.2\%) pigs in Experiment II developed antibody responses above the cut-off. Antibody levels were below cut-off in pigs \#5, 10, 16, 19 and 22 of Experiment I and pigs \#29, 43, 62, 68 of Experiment II. Of these pigs only \#43 was not shedding $L$. intracellularis after inoculation. Although the infection was cleared by antibiotic treatment (from day 21), 12/18 (66.6\%) pigs in the Re-i groups were ELISA-positive through day 49 where they were re-inoculated and after which they stayed positive with moderate to high ELISA responses for most pigs and with $15 / 18$ ( $83.3 \%)$ sero-positive pigs at termination. All pigs but one (\#17, Experiment I) in the CC groups became ELISA-positive 2-3 weeks after L. intracellularis challenge inoculation.

\subsection{WBC counts}

The effect of $L$. intracellularis infection on the total numbers of WBC in the experiments reflected the severity of the disease. In Experiment I, where the primary $L$. intracellularis infection induced clinical disease in some pigs, WBC counts $\left(10^{6} / \mathrm{ml}\right.$, mean \pm SEM) were significantly increased $(P<0.05$, Mann-Whitney $)$ in primary infected pigs at day $13(20.1 \pm 1.6$ vs. $13.9 \pm 0.9), \quad 18 \quad(21.5 \pm 0.9$ vs. $13.8 \pm 0.7)$ and $21(22.7 \pm 1.6$ vs. $16.8 \pm 1.0)$ compared 

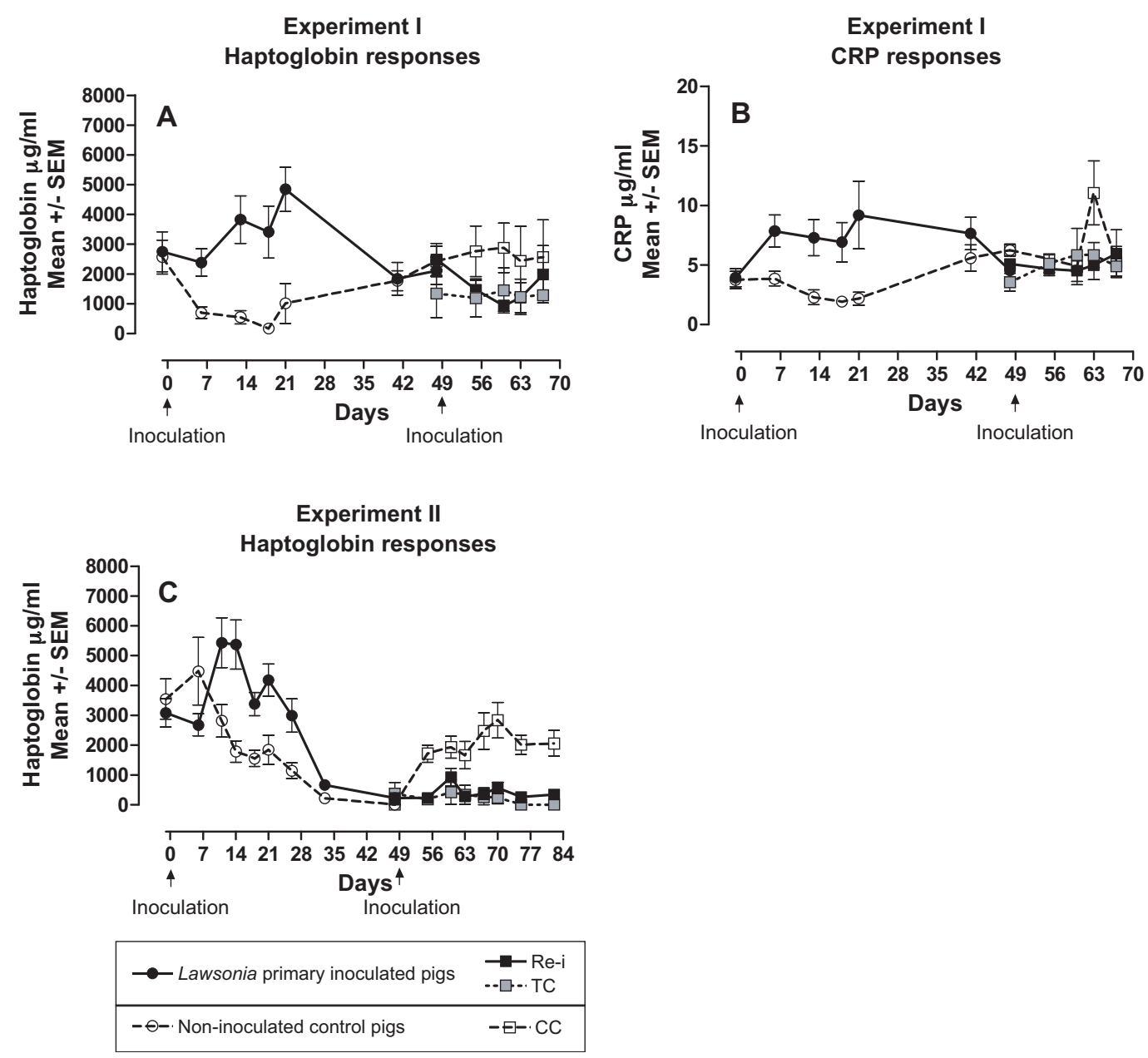

Fig. 3. Acute phase protein responses after primary L. intracellularis inoculation and challenge. The levels of haptoglobin were measured in both Experiment I (A) and II (C), whereas CRP responses were only measured in Experiment I (B). Before day 48, the TC-pigs and the Re-i pigs were grouped together as explained in the legends for Fig. 1.

to day $-1(17.1 \pm 1.1)$ and to the non-inoculated controls on the respective days. In Experiment II, where the primary infection only resulted in subclinical infections, no significant differences in levels of WBC were measured between the groups of pigs.

\section{Discussion}

Challenge inoculation of previously infected pigs did not induce faecal shedding of $L$. intracellularis, whereas pigs challenged without former $L$. intracellularis infection shed L. intracellularis from 6 days after inoculation. The present study thus clearly demonstrates that primary infected pigs are protected against re-infection with $L$. intracellularis without shedding of bacteria after the challenge inoculation. Protection against disease and shedding of $L$. intracellularis is important to prevent economic losses due to $L$. intracellularis infection and to increase pig welfare. This study therefore promotes development of effective vaccine strategies to prevent "treatment demanding L. intracellularis infection", but also to reduce the spread of $L$. intracellularis within the herd and between herds.
Collins and Love (2007) have previously reported protection with no detection of $L$. intracellularis in faecal samples of pigs challenge inoculated 10 weeks after a primary infection at three different doses (three groups with five pigs per group), whereas the challenge control group (five 4-week old pigs) showed faecal shedding of the bacteria from day 7 p.i. Our data corroborates the conclusions by Collins and Love and, as we used age matched pigs for the challenge inoculation, also excludes the possibility of an age related increase in resistance as sole explanation for their observed protection. Experimental infection with $L$. intracellularis has until now usually been done in 5-8 week old piglets (McOrist et al., 2006) and by induction of PE in the CC pigs the present data show that experimental $L$. intracellularis infection, with development of PE, can be induced in 3 month old pigs. However, although inoculation at 12 weeks of age induced PE, the severity of the infection was lower compared to inoculation at 5 weeks of age with same dose of inoculum. Thus, some age-dependent effect on pathology and level of immune responses prevail, 
and age-matched pigs must be used to compare differences between groups.

In Experiment II, we reduced the initial dose of inoculum to develop only subclinical disease in response to primary L. intracellularis infection, which still provided total protection against a challenge infection.

Statistically significant reduction in weight gain after primary $L$. intracellularis infection was observed only after inoculation of young pigs and only for the pigs in Experiment I, which were also the only pigs to experience significant changes in level of circulating leukocytes. Primary inoculation of older pigs (12-13 weeks of age) did not result in statistically significant changes in weight gain compared to the protected pigs. We did not include a group of pigs that was uninfected throughout the whole study period, and it is therefore not possible to evaluate if the stunted growth after infection in the young pigs was later compensated by increased weight after treatment.

Measurement of serum concentrations of acute phase proteins may be used to detect ongoing disease or injury caused by infective microorganisms (Petersen et al., 2004). In the pig significant increases in the serum concentrations of CRP, haptoglobin, serum amyloid A and pig major acute phase protein occur rapidly after initiation of infection (Petersen et al., 2004). The kinetics of this acute phase protein response is somewhat dependent on the infection in question (Heegaard et al., submitted for publication) and is dependent on the pathogenesis of the infection in question correlating with disturbances in tissue homeostasis. To our knowledge the current data are the first to describe the acute phase protein response to $L$. intracellularis infection in pigs. Although some variation was seen in the level of responses between pigs, significantly increased serum concentrations of haptoglobin were generally seen 2-3 weeks after inoculation, whereas CRP increased already at day 6 in the young pigs. At day 41 the concentration of haptoglobin was normalised while CRP was still elevated. Interestingly, statistically significant increased serum concentrations of haptoglobin were also observed in Experiment II in which the pigs were only subclinically infected. A significant rise in haptoglobin concentrations was also observed after primary inoculation of the older CC group, albeit smaller than seen during the primary L. intracellularis infection of young pigs while increased haptoglobin and CRP concentrations were not seen in the group of Re-i pigs. The acute phase protein responses thus reflected both the observed protection against $L$. intracellularis infection upon secondary challenge and that increased resistance to the infection develops with age. Measurement of increased haptoglobin and/or CRP may be used in combination with serological and bacteriological diagnostics to identify "treatmentdemanding-disease" caused by L. intracellularis.

Most of the pigs became sero-positive 2-3 weeks after primary inoculation, but in both experiments some pigs were slow responders with a positive response not measured before day 48 p.i., a time where the pigs had been cleared for the infection by antibiotic treatment. Irrespectively of the previous serological status of the Re-i pigs all of them were protected against re-infection as evidenced by absence of $L$. intracellularis shedding after challenge. In addition, nearly half of the pigs (45.6\%) from Experiment II had acquired $L$. intracellularis specific maternal antibodies, which were not protective against the primary infection in these pigs as evidenced by faecal shedding and increased haptoglobin concentrations after inoculation. These data therefore indicate that passively or actively acquired antibodies against $L$. intracellularis are not of significant importance in the observed protection against $L$. intracellularis re-infection.

In conclusion, we observed a distinct and reproducible protection against re-infection with experimental $L$. intracellularis infection in pigs. This protection was identified by the absence of faecal shedding of $L$. intracellularis after challenge, low detection of $L$. intracellularis (low IHC scores) at post mortem examination and a lack of an acute phase protein response after challenge in the previously infected pigs compared to the age matched challenge control pigs. Development of a protective immune response to a mucosal intracellular infection is not surprising and other studies have previously indicated this is true also for $L$. intracellularis. The current study is, however, the first to include all relevant control groups, whereby a partial age-related resistance to the infection is also documented, and to demonstrate the acute phase protein response in serum as a sensitive indicator of the level of infection. Further studies to characterize the nature of the protection are warranted for a better understanding of protective immune mechanisms and rational development of more effective vaccines against L. intracellularis infection.

\section{Acknowledgements}

The project was co-financed by The Danish Food Industry Agency (3401-65-03-745) and National Veterinary Institute. The pigs were housed at National Veterinary Institute in Copenhagen and the technicians at DTU-VET are greatly thanked for the daily care and practical participation in animal procedures. Jeanne Toft Jakobsen and Lien T. M. Nguyen are thanked for excellent technical assistance.

\section{References}

Boesen, H.T., Jensen, T.K., Jungersen, G., Riber, U., Boye, M., Møller, K., 2005a. Development, characterization and diagnostic application of a monoclonal antibody specific for a proteinase $\mathrm{K}$ resistant Lawsonia intracellularis antigen. Vet. Microbiol. 105, 199-206.

Boesen, H.T., Jensen, T.K., Møller, K., Nielsen, L.H., Jungersen, G., 2005b. Evaluation of a novel enzyme-linked immunosorbent assay for serological diagnosis of porcine proliferative enteropathy. Vet. Microbiol. 109, 105-112.

Boesen, H.T., Jensen, T.K., Schmidt, A.S., Jensen, B.B., Jensen, S.M., Møller, K., 2004. The influence of diet on Lawsonia intracellularis colonization in pigs upon experimental challenge. Vet. Microbiol. 103, 35-45.

Collins, A.M., Love, R.J., 2007. Re-challenge of pigs following recovery from proliferative enteropathy. Vet. Microbiol. 120, 381-386.

Eckersall, P.D., Conner, J.G., Parton, H., 1989. An enzyme-linked immunosorbent assay for canine C-reactive protein. Vet. Rec. 124, 490-491.

Heegaard, P.M., Pedersen, H.G., Jensen, A.L., Boas, U., 2009. A robust quantitative solid phase immunoassay for the acute phase protein C-reactive protein (CRP) based on cytidine 5'-diphosphocholine coupled dendrimers. J. Immunol. Methods 343, 112-118.

Heegaard, P.M.H., Stockmarr, A., Piñeiro, M., Carpintero, R., Lampreave, F., Campbell, F.M., Eckersall, P.D., Toussaint, M.J.M., Gruys, E., Sorensen, N.S., submitted for publication. Optimal combinations of acute phase proteins for detecting infectious disease in pigs. Vet. Res. 
Hjulsager, C.K., Grau-Roma, L., Sibila, M., Enøe, C., Larsen, L., Segalés, J., 2009. Inter-laboratory and inter-assay comarison for quantification of PCV2 nucleic acid extracted from field samples. Vet. Microbiol. 133, 172-178.

Jacobson, M., Fellström, C., Jensen-Waern, M., 2010. Porcine proliferative enteropathy: an important disease with questions remaining to be solved. Vet. J. 184, 264-268.

Jensen, T.K., Møller, K., Leser, T.D., Jorsal, S.E., 1997. Comparison of histology, immunohistochemistry and polymerase chain reaction for detection of Lawsonia intracellularis in natural porcine proliferative enteropathy. Eur. J. Vet. Pathol. 3, 115-123.

Jensen, T.K., Vigre, H., Svensmark, B., Bille-Hansen, V., 2006. Distinction between Porcine Circovirus Type 2 enteritis and porcine proliferative enteropathy caused by Lawsonia intracellularis. J. Comp. Path. 135, 176-182.

Kroll, J.J., Roof, M.B., McOrist, S., 2004. Evaluation of protective immunity in pigs following oral administration of an avirulent live vaccine of Lawsonia intracellularis. Am. J. Vet. Res. 65, 559-565.

Lindecrona, R.H., Jensen, T.K., Andersen, P.H., Møller, K., 2002. Application of a $5^{\prime}$ nuclease assay for detection of Lawsonia intracellularis in fecal samples from pigs. J. Clin. Microbiol. 40, 984-987.
McOrist, S., Gebhart, C.J., 2006. Proliferative enteropathies. In: Straw, B.E., Zimmerman, J.J., D'Allaire, S., Taylor, D.J. (Eds.), Diseases of Swine. ninth ed. Blackwell Publishing, Ames, IA, pp. 727-738.

McOrist, S., 2005. Defining the full costs of endemic porcine proliferative enteropathy. Vet. J. 170, 8-9.

McOrist, S., Gebhart, C.J., Bosworth, B.T., 2006. Evaluation of porcine ileum models of enterocyte infection by Lawsonia intracellularis. Can. J. Vet. Res. 70, 155-159.

McOrist, S., Smits, R.J., 2007. Field evaluation of an oral attenuated Lawsonia intracellularis vaccine for porcine proliferative enteropathy (ileitis). Vet. Rec. 161, 26-28.

Petersen, H.H., Nielsen, J.P., Heegaard, P.M., 2004. Application of acute phase protein measurements in veterinary clinical chemistry. Vet. Res. 35, 163-187.

Sørensen, N.S., Tegtmeier, C., Andresen, L.O., Pineiro, M., Toussaint, M.J., Campbell, F.M., Lampreave, F., Heegaard, P.M., 2006. The porcine acute phase protein response to acute clinical and subclinical experimental infection with Streptococcus suis. Vet. Immunol. Immunopathol. 113, 157-168. 